\title{
Neurological complication in HIV
}

\author{
Jagadeeswaran $\mathrm{M}^{1 *}$, Vikashini. $\mathrm{S}^{2}$, Saravanan $\mathrm{R}^{3}$, \\ Muthumariyammal. $\mathrm{V}^{4}$ \\ ${ }^{1}$ Professor, Department of Pharmaceutical Analysis, Nandha College of Pharmacy, \\ Erode, Tamilnadu, Email: jaganmurugesh@gmail.com \\ ${ }^{2}$ Assistant Professor, Department of Pharmacy Practice, Nandha College of Pharmacy, \\ Erode, Tamilnadu, Email: vikashini@nandhapharmacy.org \\ ${ }^{3}$ Associate Professor, Department of Pharmacology, Nandha College of Pharmacy, \\ Erode, Tamilnadu, Email: saravanan@nandhapharmacy.org \\ ${ }^{4}$ Assistant Professor, Medical Surgical Nursing, Nandha College of Nursing, Erode, \\ Tamilnadu, Email:Muthusweety94@gmail.com \\ ${ }^{*}$ Corresponding Author
}

\begin{abstract}
The Human Immunodeficiency Virus (HIV) is equally neurotropic as well as immune tropic, initiating severe damage on the way to both systems. Despite the fact that their number has decreased, neurological manifestations and HIV consequences are nevertheless widespread in the epoch of amalgamation antiretroviral therapy. Voguish advanced HIV illness, neurological opportunistic infections (OI) arise. Neurological problems directly connected to HIV can develop at any stage of the disease's progression, from AIDS concomitant dementia to the sterilized meningitis of original HIV contamination seen in resistant compromised people. Medicines can assist persons living with HIV in preventing the disease from progressing to AIDS. Controlling HIV can also reduce your risk of developing HIV-related nervous system disorders. The purpose of this article is to investigate the characteristics of neurological problems in HIV/AIDS patients in addition their relationship to highly active antiretroviral therapy(HAART).
\end{abstract}

Keywords: HAART, Neurological complication, HIV-1

\section{Introduction}

HIV can affect the neurological system at numerous intensities, commencing the brain to the exterior nerve also muscle, primary cutting-edge the progress of the infection. Infection can be situated classified as primary when the pathological alterations are caused solely by HIV, otherwise tributary when adaptable infections are present. Neurological illnesses are common in people infected with the human immunodeficiency virus type 1 (HIV-1) and have a substantial control on their functional capacity, quality of life, and survival. HIV infection has three stages: acute initial infection, asymptomatic (latent) infection, and symptomatic chronic illness. Disease advance is greatly varied, ranging starting 6 months following sero conversion to more than 20-30 years, with exceptional suppressors showing minimal advancement. As HIV and AIDS wreak havoc on your immune system, your central nervous system suffers as well. Both HIV and AIDS produce a variety of nervous system (neurological) issues (complications). This is especially true if an individual's HIV infection has progressed to AIDS, the most severe stage of HIV infection. Many cells in the body, including those in your nervous system, can be infected directly by HIV. However, it has a far more significant effect, notably on the nervous system, in that it induces extreme inflammation in your body. This inflammation has the potential to affect the spinal cord and brain. Nervous system problems do not usually appear until HIV has progressed. This is frequently the case when you have AIDS. Approximately $50 \%$ of individuals with AIDS have HIVrelated nervous system disorders.

\section{Complication in central nervous system (CNS)}

From the time when the start of the AIDS endemic, at hand has be located a significant escalation in Central Nervous System (CNS) Lymphoma. The vast majority of cases of major CNS lymphoma (PCNSL) transpire from time to time in people by way of certainly not obvious immunological deficit. Patients appear with altered mental status as well as local neurogenic complaints. Neurologic deficits frequently progress quickly, and the conclusion comes within 2 to 3 months. Seizures are a lesser aggregate of common in patients with gliomas. Subversion of lymphoma into the next vitric and retina. HAART devours partaken a significant impact on persistence in PCNSL patients also be duty-bound to be shadowed vigorously. Advanced dementia, vision forfeiture, paroxysmal 
quadriparesis, and ataxia are all clinical characteristics. EEG, MRI Scan, and CSF were used to make the diagnosis. The EEG revealed a gradual decrease of activity in both hemispheres. On T2weighted imaging, the MRI scan revealed significant white matter abnormalities by consensus. The presence of virus DNA in the CSF through polymerase chain rejoinder or brain surgery confirms the diagnosis. The addition of intravenous cytarabine to the treatment enhanced survival. Some diseases, such as toxoplasma encephalitis and cryptococcal meningitis, progress quickly, whereas others, such as PML and CNS lymphoma, progress slowly, frequently lasting weeks to months. However, there are several exceptions to these trends. Although many CNS opportunistic infections cause nonspecific warning sign such as fever and fatigue, the amalgamation of signs such as a new arrangement of headache or headache lasting more than 3 days, new-onset seizures, or decreased cerebral utility stalwartly suggests a grave crucial brain abrasion.

\section{Infection in nervous system caused by HIV}

In all persons with radical HIV infection, CNS adaptable infections should be suspected. Individuals who are HIV-positive but are heedless of their position may paramount extant with CNS adaptable infections. As soon as the CD4-cell count is less than 200 cells per $\mathrm{L}$, various infections are more likely to occur.

\section{Dementia}

HIV/AIDS affects several organ systems in the body, together with the brain besides neurological structure. Utmost individuals are unaware that HIV infection marks its approach to the brain primary in the illness progression. HIV encephalopathy is caused by an infection that banquets to the brain. It is one of the causes of dementia in HIV-infected patients. The more infection spreads in the brain, the shoddier the dementia signs grow.

AIDS dementia is furthermore notorious as HIV accompanying dementia or AIDS dementia intricate. It is a significant side effect of HIV infection and is customarily found in the later steps of the illness. Encephalopathy occurs when HIV spreads to the brain. The more infection spreads in the brain, the shoddier the dementia signs grow. The following are some of the symptoms connected with HIV-related dementia. Encephalitis is a disorder in which the brain and spinal column membranes enlarge. Memory lapse, Striving concentrating or continuing focused, Struggle speaking undoubtedly or precisely, Gradual loss of motor abilities, or diminished coordination are all symptoms of cognitive impairment. Examining and evaluating patients is critical in detecting the presence and amount of dementia. Indicative dealings for dementia include a mental status test, simple trials of physical facilities or crusade, magnetic resonance imaging $(\mathrm{MRI})$, computed tomography scan (also known as a CT or CAT scan), and a spinal fluid test, in tallying to a far-reaching medical history and all-embracing neurological motor and sensory exam.

The most prevalent neurological opportunistic infection is toxoplasmic encephalitis. Since toxoplasmic encephalitis is began by revival of encysted bradyzoites quite than preliminary infection, the prevalence varies affording to the population's seroprevalence of Toxoplasma gondii. The degree of immunosuppression and whether or not prophylaxis for Pneumocystis jiroveci pneumonia is utilised as risk factors for toxoplasmic encephalitis, as trimethoprim is also an effective preventative medication for toxoplasmic encephalitis. Clinical and radiological improvements following empirical treatment are frequently used to confirm the diagnosis of toxoplasmic encephalitis.

\section{Viral infections}

HIV can put you at risk for a variety of viral diseases that affect the nerve system. Infections with Cytomegalovirus can impair your capacity to think. They can also have an impact on your ability to control your leg motions and bladder. They can also impair vision, hearing, and respiration, as well as induce pneumonia. People suffering from AIDS are more susceptible to get a herpes virus infection, such as shingles. They are also more prone to brain and spinal cord inflammation. A virus can also cause progressive multifocal leukoencephalopathy (PML). PML is a highly lethal and aggressive virus. Controlling it with HIV medicines is sometimes possible. The overview of antiretroviral medication stemmed in a significant drop in cytomegalovirus infection. Whether the incidence, clinical features, and prognosis of CMV in HIV-infected patients have altered over time. CMVI is described as the isolation of a virus or the identification of viral proteins (antigens) or nucleic acid in anyone, fluid, or tissue CMV disease is divided into end-organ disease.The incidence of adequate irrefutable indications and/or signs, as well as documentation of CMV in tissue from the relevant organ by histology, virus isolation, fast culture, immunohistochemistry, or DNA hybridization, are necessary to define "confirmed CMV endorgan disease.

\section{Infections by fungi and parasites}

A fungus causes cryptococcal meningitis. It causes severe spinal cord and brain inflammation. Toxoplasma encephalitis is an infection caused by a parasite. It frequently causes confusion, seizures, and excruciating headaches. Both of these infections are potentially fatal. Cryptococcus neoformans is an encapsulated yeast that is typically found in soil and pigeon faeces. Cryptococcus gattii is a tropical and subtropical pathogen that causes illness in immunocompetent people. Meningitis is a serious medical condition that causes inflammation of the protective membranes that cover the brain and spinal cord, known collectively as the meninges. 
Meningococcus, Streptococcus pyogenes (bacterial species causing bacterial meningitis: BM), Mycobacterium tuberculosis (Tubercular meningitis: TBM), and Cryptococcus neoformans are the most common causes of meningitis (fungal meningitis: CM).People with advanced HIV are more likely to suffer from nerve damage. The virus causes nerve damage throughout the body. This causes severe pain or weakness.

\section{Lymphomas}

The incidence of lymphoma among HIV patients far outnumbers that of the all-purpose populace. The amplified menace for lymphoma give the impression to be related to a number of factors, containing the retrovirus's renovating chattels, the disease's immunosuppression and cytokine dysregulation, and, most outstandingly, resourceful infections with other lymphotrophic herpes viruses such as Epstein-Barr virus and human herpesvirus.

\section{Diagnosis of nerve effect}

HIV and AIDS can be diagnosed with a blood test. Other tests, however, will be required to examine the various sections of the neurological system and diagnose issues. Typical tests include: The electrical activity of the muscles and nerves is measured using electromyography and nerve conduction studies. A biopsy examines a sample of tissue in order to detect malignancies in the brain or inflammation in the muscles. The brain structures are photographed using radio waves and powerful magnets during an MRI test. It has the ability to detect brain inflammation, infections, tumours, strokes, and damaged tissue inside the brain and spinal cord. A spinal tap (lumbar puncture) is a procedure that collects a sample of cerebrospinal fluid to check for infections, haemorrhage, or other disorders affecting the spinal cord or brain. A CT scan exam creates a 3-D image of the brain using $\mathrm{X}$-rays and a computer. This test provides less information than an MRI.

\section{Treatment}

Medicines are used to prevent HIV from wreaking havoc on the body. They are also used to assist reduce the chance of HIV causing nervous system harm. Each nervous system disorder is dealt with in a unique way. Chemotherapy and radiation therapy can be used to treat cancer. Antibiotics are required for bacterial infections. Certain medications may be beneficial in the treatment of viral infections or nerve discomfort. Some of the mental health issues associated with HIV may be treated with counselling and antidepressant medication.

\section{Conclusions}

Cryptococcal meningitis, primary cerebral lymphoma, progressive multifocal encephalopathy, and cerebral toxoplasmosis are examples of neurological opportunistic infections and illnesses that can emerge in advanced HIV disease. Neurological disease directly connected to HIV can be discovered at any stage of the disease's progression, from AIDS-associated dementia to the aseptic meningitis of original HIV infection seen in immunocompromised people. NonHIV-related neurological illness is becoming more common in the era of efficient antiretroviral therapy, where peripheral HIV viral replication is generally controlled. Cortical atrophy is visible on cranial CT and MRI, although the degree of atrophy has not been linked to dementia severity.

\section{References}

1. Antinori A, Larussa D, Cingolani A, et al. Prevalence, associated factors, and prognostic determinants of AIDS-related toxoplasmic encephalitis in the era of advanced highly active antiretroviral therapy. Clin Infect Dis 2004; 39: 1681-91.

2. Pereira-Chioccola VL, Vidal JE, Su C. Toxoplasma gondii infection and cerebral toxoplasmosis in HIVinfected patients. Future Microbiol 2009; 4: 1363-79.

3. Berger JR, Pall L, Lanska D, Whiteman M. Progressive multifocal leukoencephalopathy in patients with HIV infection. J Neurovirol 1998; 4: 59-68.

4. Engsig FN, Hansen $A B$, Omland $L H$, et al. Incidence, clinical presentation, and outcome of progressive multifocal leukoencephalopathy in HIV-infected patients during the highly active antiretroviral therapy era: a nationwide cohort study. J Infect Dis 2009; 199: 77-83.

5. Haldorsen IS, Krakenes J, Goplen AK, et al. AIDSrelated primary central nervous system lymphoma: a Norwegian national survey 1989-2003. BMC Cancer 2008; 8: 225.

6. Bayraktar S, Bayraktar UD, Ramos JC, et al. Primary CNS lymphoma in HIV positive and negative patients: comparison of clinical characteristics, outcome and prognostic factors. J Neurooncol 2011; 101: 257-65. Anduze-Faris BM, Fillet AM, Gozlan J, et al. Induction and maintenance therapy of cytomegalovirus central nervous system infection in HIV-infected patients. AIDS 2000; 14: 517-24.

7. Holland NR, Power C, Mathews VP, et al. Cytomegalovirus encephalitis in acquired immunodefi ciency syndrome (AIDS). Neurology 1994; 44: 507-14.

8. Graybill JR, Sobel J, Saag M, et al. Diagnosis and management of increased intracranial pressure in patients with AIDS and cryptococcal meningitis. The NIAID Mycoses Study Group and AIDS Cooperative Treatment Groups. Clin Infect Dis 2000; 30: 47-54.

9. van der Horst CM, Saag MS, Cloud GA, et al. Treatment of cryptococcal meningitis associated with the acquired immunodefi ciency syndrome. National Institute of Allergy and Infectious Diseases Mycoses Study Group and AIDS Clinical Trials Group. N Engl J Med 1997; 337: 15-21. 